\title{
Effect of rat common carotid artery occlusion on vascular action of adenosine
}

\author{
Miroslav Radenković ${ }^{*}$, Radmila Stevanović $^{2}$, Marko Stojanović ${ }^{1}$ Mirko Topalović ${ }^{1}$, Milica Stojiljković ${ }^{1}$ \\ From 16th Scientific Symposium of the Austrian Pharmacological Society (APHAR) \\ Vienna, Austria. 25-27 November 2010
}

\section{Background}

Adenosine is a purine nucleoside that contributes to regulation of vascular tone in different blood vessels, especially in pathological conditions with ischemia and subsequent hypoxia. Prior studies also suggested that relaxations induced by adenosine could be dependent upon the presence of vascular endothelium and activation of various potassium channels. In regard to the previous facts the aim of this study was to investigate effect of adenosine on isolated rat common carotid artery submitted to occlusion.

\section{Materials and methods}

The current study involved three groups of Wistar rats (220-280 g): non-operated (A), sham-operated (B) and operated animals in which carotid arteries were occluded for 45 minutes (C). Anaesthesia was induced by a single i.p. application of $25 \%$ urethane. Carotid arteries were extracted from rats, carefully dissected from surrounding tissue, cut into $4 \mathrm{~mm}$ long rings and placed in an organ bath. The endothelium was removed from some rings by gently rubbing the intimal surface with stainless-steel wire. Apart from the pharmacological verification, the presence of endothelial cells was confirmed by histological evaluation on randomly selected preparations. Concentration-response curves for adenosine were obtained in a cumulative fashion on serotonin-precontracted arteries.

\section{Results}

Adenosine (0.01-100 $\mu \mathrm{M})$ produced a concentrationdependent relaxation of carotid artery with similar maximal effects in all three groups (A: $97.0 \pm 5.8 \%$; B: $99.4 \pm 2.3 \%$;
C: $102.5 \pm 2.5 \%)$. Endothelial denudation did not affect obtained maximal responses to adenosine in any of investigated clusters (A: $108.4 \pm 3.8 \%$; B: $98.5 \pm 2.0 \%$; C: $95.8 \pm$ $2.8 \%)$. In the presence of high $\mathrm{K}^{+}(100 \mathrm{mM})$ maximal relaxant responses of carotid artery from non-operated and sham-operated animals were reduced by $70.7 \%$ and $81.9 \%$, respectively, whereas after the artery occlusion the control effect produced by adenosine was reduced only by $16.6 \%$.

\section{Conclusions}

Adenosine induced endothelium-independent relaxation of carotid artery irrelevant of artery occlusion. Conversely, it appears that in a pathological setting with ischemia the signal mechanism of potassium channel activation is significantly reduced.

\section{Acknowledgements}

The Ministry of Science, Serbia, supported this research with grant 145015B,

\section{Author details}

'Department of Pharmacology, Clinical Pharmacology and Toxicology, Medical Faculty, University of Belgrade, 11129 Belgrade, Serbia. ${ }^{2}$ Institute of Pathology, Medical Faculty, University of Belgrade, 11000 Belgrade, Serbia.

Published: 16 November 2010

doi:10.1186/1471-2210-10-S1-A2

Cite this article as: Radenković et al.: Effect of rat common carotid artery occlusion on vascular action of adenosine. BMC Pharmacology 2010 10(Suppl 1):A2.

\footnotetext{
* Correspondence: mradenkovic@med.bg.ac.rs

'Department of Pharmacology, Clinical Pharmacology and Toxicology,

Medical Faculty, University of Belgrade, 11129 Belgrade, Serbia

Full list of author information is available at the end of the article
} 